\title{
Common peroneal nerve schwannoma, a rare cause of sciatica - Case report $\&$ review of literature
}

\author{
Iroshani Kodikara', Dhanusha Gamage ${ }^{2}$ \\ 'Department of Anatomy, Faculty of Medicine, University of Ruhuna, Galle \\ ${ }^{2}$ Base Hospital, Tissamaharama.
}

\section{Introduction}

Schwannoma typically a slow-growing tumour that bears the reputation of the most common benign peripheral nerve sheath tumour. Sciatic nerve schwannoma is rare (1\%) tumour that can manifest with symptoms of sciatica (1). Diagnosis of schwannoma is often delayed, notably when presents with symptoms of sciatica (1-4).

Keywords: common peroneal nerve shwannoma, sciatica, neurofibroma, malignant peripheral nerve sheath tumours.

\section{Case presentation}

We report a case of common peroneal nerve schwannoma in a 53-year-old man presented with left leg pain for two years duration. He complained of severe and intermittent pain in the posterior aspect of the left calf, which progressed over the time. Though the pain was more apparent on walking, the characteristic claudication distance was not elicited. He experienced a shooting pain when squatting down or sits on a high chair. However, the pain was not exaggerated on coughing or trunk movements. He was apparently well before and was a non-smoker and a teetotaler.
On examination, he was average built; general and systemic examination was unremarkable with no definite neurological signs. The tendon reflexes were normal, and the movement range of the affected lower limb was normal. The straight leg raising test was negative. No chronic ulcers or trophic changes were found in the lower limbs.

In the process of investigations, to exclude a vascular pathology, he referred to the Radiology department for lower limb arterial and venous doppler studies. The arterial and venous doppler studies were not diagnostic of an arterial or venous pathology. However, a well-defined spindleshaped mass in the left popliteal fossa was detected incidentally, which measured $3 \times 2 \times 1.5 \mathrm{~cm}$ in maximum craniocaudal, transverse and anteroposterior diameters respectively. The mass had a hypoechoic periphery with a relatively echogenic centre; minimal internal vascularity and posterior acoustic enhancement (Fig.1).

Medial to the mass was the left posterior tibial nerve that maintained normal morphology and thickness. Posteromedial to the mass were the popliteal artery and the vein, which were not compressed by the mass (Fig.3). 
Iroshani Kodikara, Dhanusha Gamage - Common peroneal nerve schwannoma, a rare cause of sciatica - Case report $\&$ review of literature

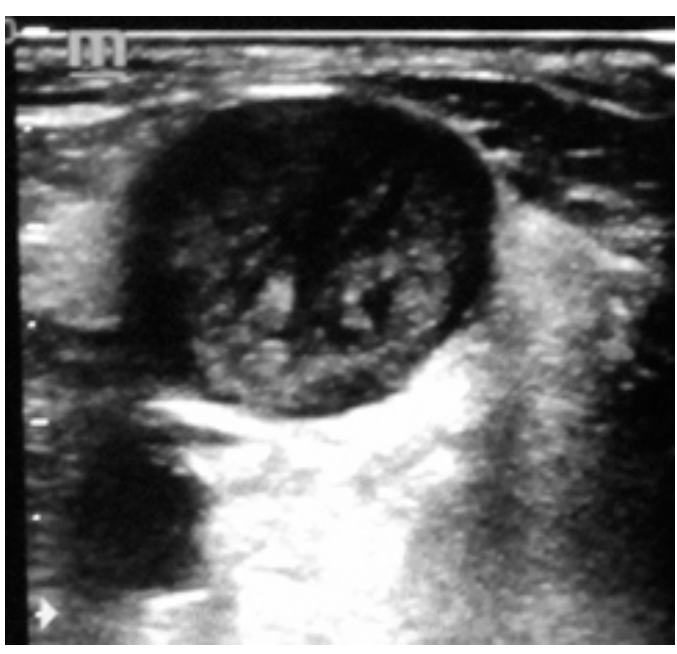

Fig. 1: Target sign

Eccentrically, it continued with the left common peroneal nerve (Fig.2).

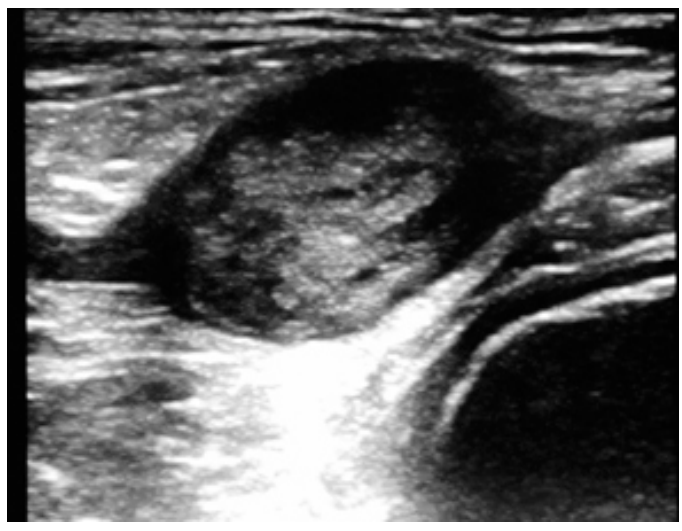

Fig. 2: Eccentric peripheral nerve continuity of the lesion

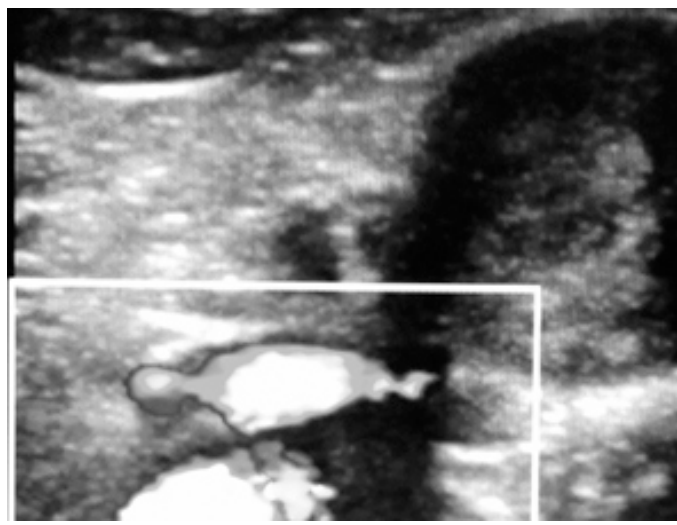

Fig. 3: Relationship of popliteal artery, vein and posterior tibial nerve to the lesion
(Artery and veins are indicated in the white box, nerve is indicated by the white arrow)

A peripheral nerve sheath tumour (PNST) of the left common peroneal nerve, causing sciatica-like symptoms was diagnosed, considering the location, ultrasound features and the relationship to the nerve. Once the diagnosis was made, the patient examined further to elicit specific signs such as the "Tinnel sign". The "Tinnel sign" was positive, which is a radiating pain along the left leg when percussing on the mass, confirming the neural origin.

A few weeks later, the mass excised surgically, upon which the symptoms disappeared. The histopathological report confirmed the diagnosis as a schwannoma of the left common peroneal nerve.

\section{Discussion}

Chronic leg pain is a common symptom that usually attributed to neural/ vascular origin or loco-regional pathologies. Detecting the aetiology of pain in our patient was tricky owing to non-specific symptoms; the symptoms were not compatible with neither the typical intermittent claudication pain of arterial origin nor the radicular type of pain of neurogenic origin. The radicular type of leg pain is typically attributed to a degenerative spinal pathology, hence even in the presence of typical pain, diagnosing a schwannoma is often delayed. Therefore, a whole limb imaging study, preferably an MRI scan 
Iroshani Kodikara, Dhanusha Gamage - Common peroneal nerve schwannoma, a rare cause of sciatica - Case report $\&$ review of literature

recommended for patients, who are having the characteristic symptoms of neural pathology while the preliminary investigation findings rule out any degenerative spinal pathology $(5,6)$. Nevertheless, in our case, the benign peripheral nerve sheath tumour (PNST) was incidentally diagnosed during a vascular doppler study.

Schwannomas are tumours of Schwann cells, which form the myelin sheath around the nerve axons. Schwannomas are known to occur in cranial \& peripheral nerves. However, the sympathetic nervous system involvement is rare (1-4). The nerve sheath tumours could be solitary or multiple; benign or malignant.

Schwannoma and neurofibroma recognized as a common benign solitary nerve sheath tumour (7) - the schwannoma considered to be the commonest benign PNST. The common peroneal nerve schwannoma reported earlier in the literature (8).

Differentiation between benign PNST from a malignant PNST is difficult with cross-sectional imaging, particularly with sonography (USS). Superficial PNST are amenable to USS assessment than the deep-seated PNST. The USS features of PNST are variable; commonly a low homogenously echogenic solid mass with posterior acoustic enhancement and positive "target sign". The continuity to a peripheral nerve is the pathognomonic feature of neural origin, can be demonstrated sonographically. Schwannomas are eccentrically related to a peripheral nerve. However, sonographic differentiation of PNST from a cystic lesion such as uncomplicated ganglion cyst is often tricky, hence misdiagnosis is frequent. Both PNST and cystic lesions are low echogenic lesions with posterior acoustic enhancement; the internal vascularity is a feature (in a colour doppler imaging) of a solid lesion, while avascularity is typical in the cystic masses (9).

Differentiating neurofibroma from schwannoma is possible only to a certain extent when imaged with USS \& MRI; neurofibroma shows a central communication to the nerve while the schwannoma is eccentrical. However, the differentiation between benign from malignant PNST is not successful even with MRI. Usually, with MRI, the benign entity is homogeneous, isointense to the muscles in $\mathrm{T} 1$ and hyperintense in T2-weighted images. In contrast, the malignant entity is inhomogeneous bearing haemorrhagic and necrotic areas. Presence of aggressive imaging features such as indistinct margins, infiltration and invasion of the surrounding tissues, favours the diagnosis of a malignant lesion.

MRI is better than USS to delineate imaging features of PNST such as

"'target sign", "fascicular sign" and "split fat sign". The "split fat sign" is the presence of a thin rim of fat around the nerve sheath tumour (Fig. 4). 
Iroshani Kodikara, Dhanusha Gamage - Common peroneal nerve schwannoma, a rare cause of sciatica - Case report $\&$ review of literature

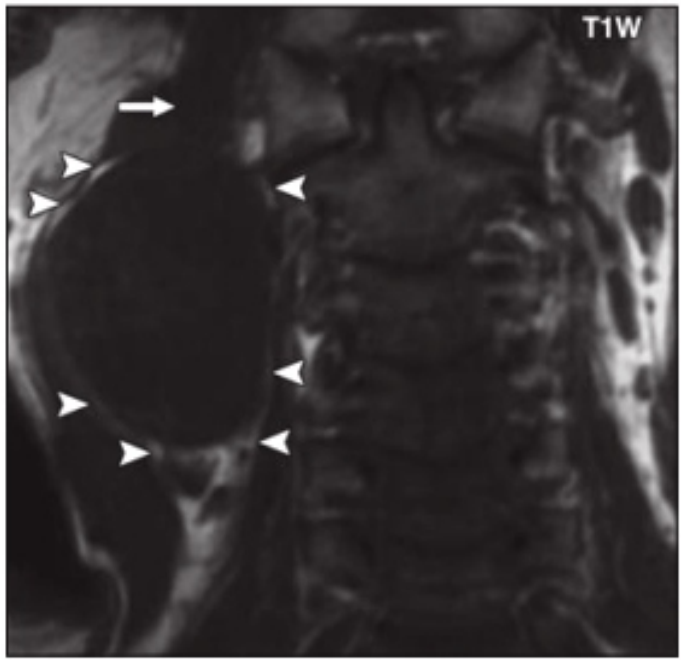

Fig. 4: Split fat sign (11)

(Thin fat rim around the lesion is indicated with arrow heads)

The "fascicular sign" (Fig. 5) described in T2 weighted images as multiple low signal intensities surrounded by a hyperintense rim, which probably represents nerve fascicles $(10,11)$.

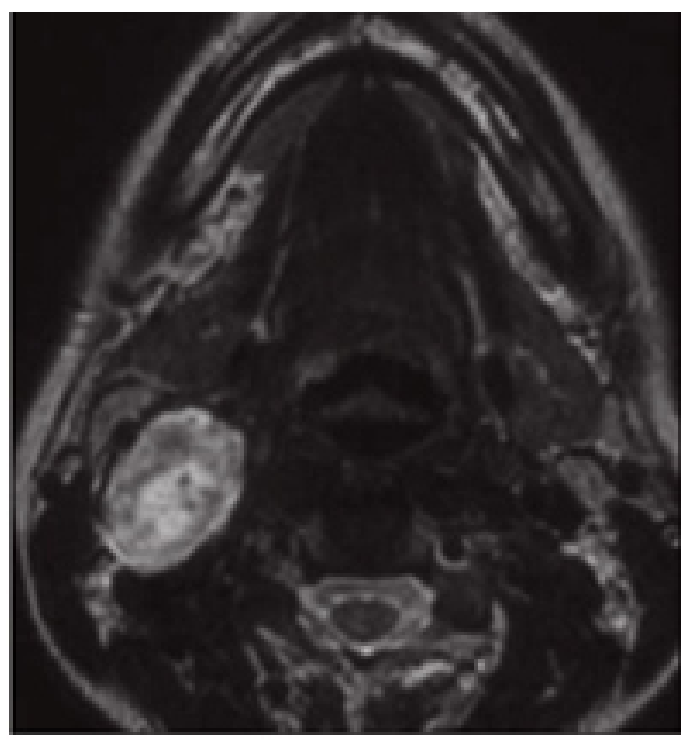

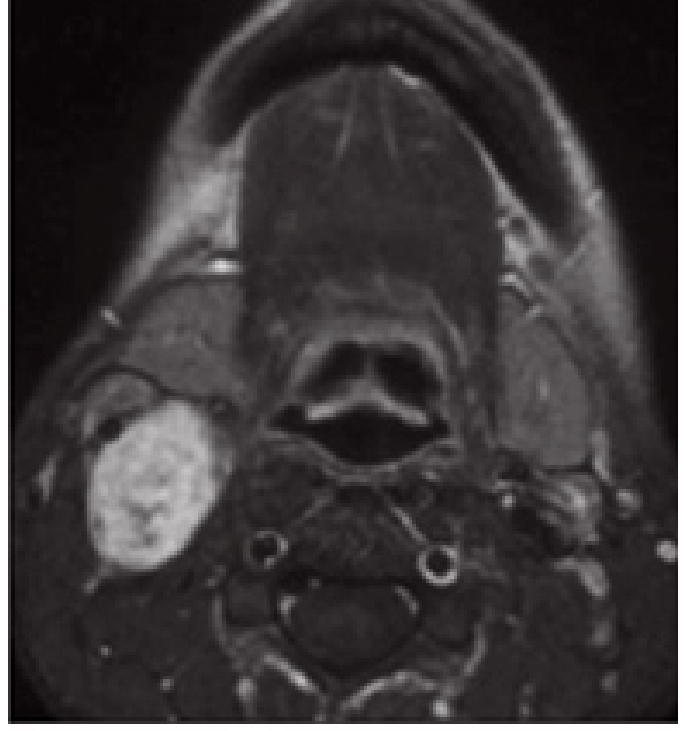

Fig. 5: Fascicular sign (11)

(multiple low signal intensity surrounded by hyper intense rim in T2 weighted images)

The "target appearance" of PNST is due to concentrically arranged Antony type A \& B bodies: Antony type-A bodies are peripheral, and Antony type$\mathrm{B}$ bodies are central. Computed tomography (CT) does not provide an added advantage over MRI in differentiating schwannoma from neurofibroma; in fact, the MRI is better than CT. The CT appearances of PNST often include uniformly low-attenuated tumour mass with the "target appearance" that is a hypoattenuating centre with a hyperattenuating periphery (11).

Malignant transformation of benign PNST said to be rare. Benign and malignant imaging features and basic demographic features of PNST are described in Table 1 (10). 
Iroshani Kodikara, Dhanusha Gamage - Common peroneal nerve schwannoma, a rare cause of sciatica - Case report $\&$ review of literature

Table 1: Typical demographic and radiological features of nerve sheath tumours (10)

\section{Feature}

\section{Tumour type}

\begin{tabular}{|c|c|c|c|}
\hline & Shwannoma & Neurofibroma & Malignant PNST \\
\hline \multicolumn{4}{|l|}{ Demography } \\
\hline Prevalence & $\begin{array}{l}5 \% \text { of all benign soft } \\
\text { tissue tumours }\end{array}$ & $\begin{array}{l}5 \% \text { of all benign soft } \\
\text { tissue tumours }\end{array}$ & $6 \%$ of all sarcomas \\
\hline \multicolumn{4}{|l|}{ Affected patients } \\
\hline Age $(\mathrm{Y})$ & $25-65$ & $20-55$ & $20-65$ \\
\hline Male:female ratio & $1.3: 1$ & $1.2: 1$ & $1: 1$ \\
\hline $\begin{array}{l}\text { Multiplicity and } \\
\text { association with NF1 }\end{array}$ & $\begin{array}{l}\text { Rarely multiple; } 5- \\
18 \% \text { of patients with } \\
\text { multiple lesions have } \\
\text { NF1 }\end{array}$ & $\begin{array}{l}\text { Typically solitary, but } \\
\text { multiple when } \\
\text { associated with NF1 }\end{array}$ & $\begin{array}{l}\text { Solitary, about } 50 \% \\
\text { occurs in patients } \\
\text { with NF1 }\end{array}$ \\
\hline Malignant change & Extremely rare & $\begin{array}{l}\text { Extremely rare, } \\
\text { except in NF1 }\end{array}$ & $\begin{array}{l}\text { Seen in }<5 \% \text { of } \\
\text { patients with NF1 } \\
\text { (range } 2-29 \% \text { ) }\end{array}$ \\
\hline Lesion location & $\begin{array}{l}\text { Most often in lower } \\
\text { limbs, followed by } \\
\text { torso, upper limb and } \\
\text { retro peritoneum }\end{array}$ & $\begin{array}{l}\text { Most often in head } \\
\text { and neck, lower limbs } \\
\text { and torso followed by } \\
\text { upper limb }\end{array}$ & $\begin{array}{l}\text { Seen in major nerve } \\
\text { trunks (commonly in } \\
\text { proximal extremities } \\
\text { and torso) }\end{array}$ \\
\hline
\end{tabular}

\section{Radiologic features}

Relationship of mass to the nerve

Capsule

Target sign

Fascicular sign

Intra-tumoral cysts

Margins
Eccentric, relative to the nerve \& inseparable from nerve

$70 \%$ of cases

$50 \%$ of cases

Common

Common

Well circumscribed
Central, relative to the nerve

$30 \%$ of cases

$50-70 \%$ of cases

Common

Rare

Well circumscribed
Central, relative to the nerve \& infiltrates the nerve

Rare

Absent

Occasional, focal

Not applicable

Most often well circumscribed than irregular

(Nf1- Neurofibromatosis 1) 
Iroshani Kodikara, Dhanusha Gamage - Common peroneal nerve schwannoma, a rare cause of sciatica - Case report \& review of literature

\section{Conclusion}

Peripheral nerve sheath tumours can present as sciatica. For patients with characteristic symptoms but with negative spinal imaging studies, a whole limb MRI scan would facilitate the diagnosis of PNST. The continuation of the mass with a nerve is the pathognomonic imaging feature of neural origin. Lack of specific imaging features renders the differentiation difficult, between neurofibroma from schwannoma and benign from malignant tumour, with routine imaging such as ultrasonography and CT. Hence, MRI or biopsy remains the gold standard.

\section{Conflict of interest}

None declared.

\section{Correspondence}

Dr. Iroshani Kodikara, Senior Lecturer in Anatomy \& Consultant Radiologist, Department of Anatomy, Faculty of Medicine, University of Ruhuna, Galle, Sri Lanka.

E-mail: iroshani.kodikara@gmail.com Tel: +94 718390027

\section{References}

1. Rhanim A, El Zanati R, Mahfoud M, Berrada MS, El Yaacoubi M. A rare cause of chronic sciatic pain: Schwannoma of the sciatic nerve. J
Clin Orthop Trauma. 2013;4(2):8992. doi:10.1016/j.jcot.2013.04.001

2. Rustagi T, Badve S, Parekh AN. Sciatica from a Foraminal Lumbar Root Schwannoma: Case Report and Review of Literature. Case Rep Orthop. 2012;2012:1-3. doi:10.1155/2012/142143

3. Ninomiya K, Iwatsuki K, Ohnishi Y, Moriwaki T, Yoshimine T. Case Report A case of intrapelvic sciatic nerve schwannoma presenting as piriformis syndrome. J Neurosci Behav Heal. 2014;6(1):1-4. doi:10.5897/JNBH2014.0116

4. Anil G, Tan TY. Imaging characteristics of schwannoma of the cervical sympathetic chain: A review of 12 cases. Am J Neuroradiol. 2010;31(8):14081412. doi:10.3174/ajnr.A2212

5. Mansukhani SA, Butala RR, Shetty $\mathrm{SH}$, Khedekar RG. Sciatic nerve schwannoma : a case report. 2015;23(2):259-261.

6. Munakomi S, Shrestha P. Case Report: Sciatic nerve schwannoma a rare cause of sciatica. F1000Research. 2017;6:267. doi:10.12688/f1000research.11108. 1

7. Beaman FD, Kransdorf MJ, Menke DM. Schwannoma: radiologicpathologic correlation. Radiographics. 2004;24(5):14771481. doi:10.1148/rg.245045001

8. Hybbinette C-H. Solitary benign nerve sheath tumours around the 
Iroshani Kodikara, Dhanusha Gamage - Common peroneal nerve schwannoma, a rare cause of sciatica - Case report \& review of literature

knee joint: Report of Four Cases. Acta Orthop Scand.

1973;44(3):296-302.

doi:10.3109/174536773089886696

9. Anil G, Tan TY, Reynolds DL, Jacobson JA, Jamadar DA, Hayes CW. Sonographic Characteristics of. Am J Neuroradiol. 2010;31:1408-1412. doi:10.3174/ajnr.A2212
10. Beaman FD, Kransdorf MJ, Menke DM. Schwannoma: radiologicpathologic correlation. Radiographics. 2004;24(5):14771481. doi:10.1148/rg.245045001

11. Anil G, Tan T-Y. CT and MRI Evaluation of Nerve Sheath Tumors of the Cervical Vagus Nerve. Am J Roentgenol. 2011;197(1):195-201. doi:10.2214/AJR.10.5734 